$$
\begin{array}{lc}
{[(5), 9 .]} & r-3 s .=. i \\
{[(4),(6)]} & p-3 q .=. r-3 s \\
{[11.03]} & (7)=(1)(2) \\
{[(7),(8)]} & (1)(2) \\
{[11.2]} & (1)(2)-3(1) \\
{[12.17]} & (1)(2)-3(2) \\
{[(9),(10)]} & (1) \\
{[(9),(11)]} & (2) .
\end{array}
$$

The paradox stated above is a particular case of Theorem 10, and therefore requires no further proof.

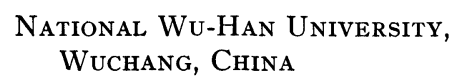

\title{
THE BETTI NUMBERS OF CYCLIC PRODUCTS
}

\author{
BY R. J. WALKER
}

1. Introduction. In a recent paper $\dagger \mathrm{M}$. Richardson has discussed the symmetric product of a simplicial complex and has obtained explicit formulas for the Betti numbers of the twoand three-fold products. Acting on a suggestion of Lefschetz, we define a more general type of topological product and apply Richardson's methods to compute the Betti numbers of a certain one of these, the "cyclic" product.

2. Basis for m-Cycles of General Products. Let $S$ be a topological space and $G$ a group of permutations on the numbers $1, \cdots, n$. The product of $S$ with respect to $G, G(S)$, is the set of all $n$-tuples $\left(P_{1}, \cdots, P_{n}\right)$ of points of $S$, where $\left(P_{i_{1}}, \cdots, P_{i_{n}}\right)$ is to be regarded as identical with $\left(P_{1}, \cdots, P_{n}\right)$ if and only if the permutation $\left(1_{i_{1} \ldots i_{n}}^{1} \ldots n_{n}\right)$ is an element of $G$. A neighborhood of $\left(P_{1}, \cdots, P_{n}\right)$ is the set of all points $\left(Q_{1}, \cdots, Q_{n}\right)$ for which $Q_{i}$ belongs to a fixed neighborhood of $P_{i}$. It is not difficult to verify that the

$\dagger$ M. Richardson, On the homology characters of symmetric products, Duke Mathematical Journal, vol. 1 (1935), pp. 50-69. We shall refer to this paper as $\mathrm{R}$. 
Hausdorff axioms hold for this definition of neighborhood, and hence that $G(S)$ is a topological space. In particular, if $G$ is the identity or the symmetric group, $G(S)$ is, respectively, the direct or the symmetric product of $S$. If $G$ is the cyclic group on $n$ elements we shall call $G(S)$ the $n$-fold cyclic product of $S$.

The space $G(S)$ can be obtained in another manner. Let $S^{n}$ denote the $n$-fold direct product of $S$. Then each element $\left(\begin{array}{l}1 \ldots \ldots n \\ i_{1} \ldots, i_{n}\end{array}\right)$ of $G$ gives rise to an automorphism of $S^{n}$ which carries $\left(P_{1}, \cdots, P_{n}\right)$ into $\left(P_{i_{1}}, \cdots, P_{i_{n}}\right)$. By identifying points which are images of each other under the group of automorphisms we evidently obtain a space homeomorphic to $G(S)$.

Now let $K$ be a simplicial complex, $K^{n}$ its direct product, and $k=G(K)$ its product with respect to the group $G$ of degree $n$ and order $r$. We then have $r$ automorphisms $T_{\lambda}$ of $K^{n}$, and a continuous, single-valued transformation $\Lambda$ of $K^{n}$ into $k$, such that $\dagger$

$$
\Lambda T_{\lambda}=\Lambda .
$$

Richardson has shown $\ddagger$ that $K^{n}$ and $k$ can be subdivided into simplexes in such a fashion that the transformations $T_{\lambda}$ and $\Lambda$ are simplicial. We can therefore operate with them on chains of $K^{n}$. If $E$ and $e$ are simplexes of $K^{n}$ and $k$, respectively, such that $e=\Lambda E$, we define the operator $\Lambda^{\prime}$ by $\Lambda^{\prime} e=\sum_{\lambda} T_{\lambda} E$. We have then

$$
\begin{aligned}
\Lambda \Lambda^{\prime} e & =r e, \\
\Lambda^{\prime} \Lambda E & =\sum_{\lambda} T_{\lambda} E .
\end{aligned}
$$

We also find that $T_{\lambda}, \Lambda$, and $\Lambda^{\prime}$ preserve boundaries and hence homologies.

The principal theorem of Richardson, $\S$ concerning the Betti numbers of $k$, is stated in terms of matrices. For actual computation we find it easier to work with the cycles themselves, and so we shall state and prove the theorem in a slightly different form.

$\dagger$ In the expression for the product of two transformations, the transformation represented by the right-hand symbol is to be applied first.

$\ddagger$ R, pp. 51 and 53 .

$\S$ R, p. 52 . 
THEOREM 1. Let $\left\{\Gamma^{i}\right\}$ be an independent basis, with respect to homology, for m-cycles, with rational coefficients, of $K^{n}$, such that $T_{\lambda} \Gamma^{i}= \pm \Gamma^{i} \lambda,(\lambda=1, \cdots, r)$; and let $\left\{\bar{\Gamma}^{\alpha}\right\}$ be a maximal subset of $\left\{\Gamma^{i}\right\}$ such that

$$
T_{\lambda} \bar{\Gamma}^{\alpha} \neq \pm \bar{\Gamma}^{\beta}, \quad(\alpha \neq \beta),
$$

for any $\lambda$. Then $\left\{\gamma^{\alpha}\right\}=\left\{\Lambda \bar{\Gamma}^{\alpha}\right\}$ is an independent basis with respect to homology for the $m$-cycles of $k$.

Proof. (i) The $\gamma^{\alpha}$ are independent. For suppose that we have $\sum_{\alpha} x_{\alpha} \gamma^{\alpha} \sim 0$, that is, $\sum_{\alpha} x_{\alpha} \Lambda \bar{\Gamma}^{\alpha} \sim 0$. Then

$$
\Lambda^{\prime} \sum_{\alpha} x_{\alpha} \Lambda \bar{\Gamma}^{\alpha}=\sum_{\alpha} x_{\alpha} \Lambda^{\prime} \Lambda \bar{\Gamma}^{\alpha}=\sum_{\alpha, \lambda} x_{\alpha} T_{\lambda} \bar{\Gamma}^{\alpha} \sim 0,
$$

by (3). Now if $T_{\lambda} \bar{\Gamma}^{\alpha}=\epsilon \Gamma^{i}, \epsilon= \pm 1$, we cannot have $T_{\mu} \bar{\Gamma}^{\alpha}=-\epsilon \Gamma^{i}$, for this would imply

$$
T_{\mu}^{-1} T_{\lambda} \bar{\Gamma}^{\alpha}=\epsilon T_{\mu}^{-1} \Gamma^{i}=-\epsilon^{2} \bar{\Gamma}^{\alpha}=-\bar{\Gamma}^{\alpha},
$$

contrary to condition (b). Similarly, from (a), we cannot have $T_{\mu} \bar{\Gamma}^{\beta}= \pm \Gamma^{i}, \beta \neq \alpha$. Hence with each such $\Gamma^{i}$ there is associated an $\epsilon_{i}$, a $\bar{\Gamma}^{\alpha}$, and $s_{i}$ values of $\lambda$ for which $T_{\lambda} \bar{\Gamma}^{\alpha}=\epsilon_{i} \Gamma^{i}$. If the last homology is now written in terms of the basis $\left\{\Gamma^{2}\right\}$, the coeffcient of $\Gamma^{i}$ will be $\epsilon_{i} s_{i} x_{\alpha}$. Since the $\Gamma^{i}$ are independent, $\epsilon_{i} s_{i} x_{\alpha}=0$, and therefore every $x_{\alpha}=0$.

Use was made of the properties of the rational coefficients only in the last step of each part of the proof. Now the $s_{i}$ introduced in (i) are factors of $r$, for the $T_{\lambda}$ for which $T_{\lambda} \bar{\Gamma}^{\alpha}=\epsilon_{i} \Gamma^{i}$ evidently form a coset of the subgroup which leaves $\bar{\Gamma}^{\alpha}$ invariant. It follows that the theorem will hold for any coefficient group in which each element has a unique $r$ th part; in particular for the group of residues modulo a number prime to $r$.

(ii) $\left\{\gamma^{\alpha}\right\}$ is a basis. We note first that since the set $\left\{\bar{\Gamma}^{\alpha}\right\}$ is maximal every $\Gamma^{i}$ is of one of the two forms $T_{\lambda} \bar{\Gamma}^{\alpha}$ or $\tilde{\Gamma}^{i}$, where for each $j$ there is a $\lambda_{j}$ such that $T_{\lambda_{j}} \tilde{\Gamma}^{j}=-\tilde{\Gamma}^{j}$. Also, $\Lambda \widetilde{\Gamma}^{j}=\Lambda T_{\lambda_{j}} \widetilde{\Gamma}^{j}$ $=-\Lambda \tilde{\Gamma}^{i}$, so that $\Lambda \tilde{\Gamma}^{i}=0$. Now if $\gamma$ is any $m$-cycle of $k, \Lambda^{\prime} \gamma$ is an $m$-cycle of $K^{n}$, and so

$$
\Lambda^{\prime} \gamma \sim \sum_{i} x_{i} \Gamma^{i}=\sum_{\alpha, \lambda} x_{\alpha \lambda} T_{\lambda} \bar{\Gamma}^{\alpha}+\sum_{i} x_{j} \widetilde{\Gamma}^{j} .
$$


Hence

$$
\Lambda \Lambda^{\prime} \gamma=r \gamma \sim \sum_{\alpha, \lambda} x_{\alpha \lambda} \Lambda T_{\lambda} \bar{\Gamma}^{\alpha}+\sum_{j} x_{j} \Lambda \tilde{\Gamma}^{j}=\sum_{\alpha, \lambda} x_{\alpha \lambda} \gamma^{\alpha}
$$

by (2) and (1). That is,

$$
\gamma \sim \sum_{\alpha, \lambda} \frac{x_{\alpha \lambda}}{r} \gamma^{\alpha}
$$

3. Betti Numbers of Cyclic Products. Keeping the notation as before, we let $G$ be the cyclic group on $n$ elements. To compute the $m$ th Betti number of the cyclic product $k$ we must count the number of $m$-cycles $\bar{\Gamma}^{\alpha}$. A basis of the type $\left\{\Gamma^{i}\right\}$ used in the theorem is obtained by taking all cycles of the form

$$
C_{m_{1}} \times \cdots \times C_{m_{n}}, \quad m_{1}+\cdots+m_{n}=m
$$

$C_{m_{i}}$ being a member of a basis of $m_{i}$-cycles of $K . \dagger$ Following Richardson's procedure, we obtain

$$
\begin{aligned}
& T_{\lambda}\left(C_{m_{1}} \times \cdots \times C_{m_{\lambda}} \times C_{m_{\lambda+1}} \times \cdots \times C_{m_{n}}\right) \\
& =(\dot{-} 1)^{\epsilon_{\lambda}} C_{m_{\lambda+1}} \times \cdots \times C_{m_{n}} \times C_{m_{1}} \times \cdots \times C_{m_{\lambda}} \text {, }
\end{aligned}
$$

where

$$
\begin{aligned}
\epsilon_{1}=m_{1} m_{2}+\cdots+m_{1} m_{n}=m_{1}\left(m-m_{1}\right) & =m m_{1}-m_{1}^{2} \\
& \equiv m m_{1}-m_{1} \quad(\bmod 2) \\
& =(m-1) m_{1},
\end{aligned}
$$

and by induction

$$
\epsilon_{\lambda} \equiv(m-1)\left(m_{1}+\cdots+m_{\lambda}\right) \quad(\bmod 2) .
$$

Let $q$ be a factor of $n, n=q s$, and consider all $\Gamma^{i}$ which are invariant, to within change of sign, under $G_{q}$, the cyclic subgroup of $G$ of order $q$. They necessarily have the form

$$
\begin{aligned}
& \Gamma_{q}=\left(C_{m_{1}} \times \cdots \times C_{m_{s}}\right) \times\left(C_{m_{1}} \times \cdots \times C_{m_{s}}\right) \times \cdots \\
& \times\left(C_{m_{1}} \times \cdots \times C_{m_{s}}\right),
\end{aligned}
$$

there being $q$ identical sets of factors. We must have $q\left(m_{1}+\right.$ 
$\left.\cdots+m_{s}\right)=m$; that is, to have a $\Gamma_{q}, q$ must be a factor of $m$ and hence of $(m, n)$, the highest common factor of $m$ and $n$. If $t$ is a proper multiple of $q$ and a factor of $(m, n)$, it is easily seen that a $\Gamma_{t}$ is also a $\Gamma_{q}$. We denote by $\Gamma_{q}^{*}$ any $\Gamma_{q}$ which is not such a $\Gamma_{t}$, and by $A_{m, q}$ the number of $\Gamma_{q}^{*}$. The total number of $\Gamma_{q}$ is then $\sum_{t} A_{m, t}$, the summation being over all values of $t$ which are multiples of $q$ and factors of $(m, n)$. But the number of $\Gamma_{q}$ is evidently equal to the number of possible combinations of the form $C_{m_{1}} \times \cdots \times C_{m_{s}}, m_{1}+\cdots+m_{s}=m / q$, and this is exactly $R_{m / q}\left(K^{s}\right)$. Hence

$$
\sum_{t} A_{m, t}=R_{m / q}\left(K^{n / q}\right)
$$

and from these equations we can obtain the $A_{m, q}$ step by step starting with $q=(m, n)$, or directly by the use of the Dedekind inversion formula.

Now

$$
T_{s} \Gamma_{q}=(-1)^{(m-1)\left(m_{1}+\cdots+m_{s}\right)} \Gamma_{q}=(-1)^{(m-1) m / q} \Gamma_{q},
$$

and so if $m$ is even and $m / q$ is odd, $\Gamma_{q}$ is a cycle of the type $\tilde{\Gamma}^{j}$ of Theorem 1 and is not counted among the $\bar{\Gamma}^{\alpha}$. We therefore put

$$
B_{m, q}=\left\{\begin{array}{l}
0, \text { if } m \text { is even and } m / q \text { is odd } \\
A_{m, q} \text { otherwise }
\end{array}\right.
$$

Consider the $s$ cycles $\Gamma_{q}^{*}, T_{1} \Gamma_{q}^{*}, \cdots, T_{s-1} \Gamma_{q}^{*}$. If any two of these are equal, say $T_{i} \Gamma_{q}^{*}=T_{j} \Gamma_{q}^{*},(i>j)$, then $\Gamma_{q}^{*}$ is invariant, to within change of sign, under the subgroup generated by $T_{j}^{-1} T_{i}=T_{i-j}$, and hence under the minimal subgroup containing $G_{q}$ and $T_{i-j}$. Since $i-j<s, T_{i-j}$ is not an element of $G_{q}$ and therefore this subgroup is a $G_{t}$ with $t$ a proper multiple of $q$, contrary to the definition of $\Gamma_{q}^{*}$. It follows that there are exactly $s=n / q$ distinct transforms of each of the $B_{m, q}$ cycles $\Gamma_{q}^{*}$, and so we can pick out $(q / n) B_{m, q}$ of the $\Gamma_{q}^{*}$ which are not transformable into one another and which can therefore be included among the $\bar{\Gamma}^{\alpha}$ of Theorem 1 . Since the cycles $\Gamma_{q}^{*}$ for different values of $q$ are not transformable into one another and since every $\Gamma^{i}$ is a $\Gamma_{q}^{*}$ for some $q$, we have the following result. 


\section{Theorem 2.}

$$
R_{m}(k)=(1 / n) \sum_{q} q B_{m, q},
$$

the summation being over all factors of $(m, n)$.

The following special cases may be of interest.

Corollary 1. If $n$ is an odd prime

$$
R_{m}(k)=\left\{\begin{array}{l}
(1 / n) R_{m}\left(K^{n}\right), \text { if }(m, n)=1, \\
(1 / n)\left[R_{m}\left(K^{n}\right)-R_{s}(K)\right]+R_{s}(K), \text { if } m=n s .
\end{array}\right.
$$

Corollary 2. If $p$ is an odd prime and $n=p^{\alpha}, m=p^{\beta} m_{1}$, $\left(m_{1}, p\right)=1$, and $\gamma=\min \alpha, \beta$,

$$
R_{m}(k)=\frac{p-1}{n}\left[\frac{1}{p-1} R_{m}\left(K^{n}\right)+\sum_{i=1}^{\gamma} p^{i-1} R_{m / p^{i}}\left(K^{n / p^{i}}\right)\right] .
$$

Corollary 3. If $R_{0}(K)=1$, then $R_{1}(k)=R_{1}(K)$.

4. Remark. The methods used on the cyclic product can evidently be used to compute the Betti numbers of a product with respect to an arbitrary group. In general, however, the resulting formulas are too complicated to be of interest.

Cornell University 